\title{
SEVEN YEARS OF REGIONAL MALARIA CONTROL COLLABORATION- MOZAMBIQUE, SOUTH AFRICA, AND SWAZILAND
}

\author{
BRIAN L. SHARP, IMMO KLEINSCHMIDT,* ELISABETH STREAT, RAJENDRA MAHARAJ, KAREN I. BARNES, \\ DAVID N. DURRHEIM, FRANCES C. RIDL, NATASHA MORRIS, ISHEN SEOCHARAN, SIMON KUNENE, \\ JACOBUS J. P. LA GRANGE, JOTHAM D. MTHEMBU, FRANCOIS MAARTENS, CARRIN L. MARTIN, AND \\ AVERTINO BARRETO
}

Malaria Research Programme, Medical Research Council, Durban, South Africa; Maputo Province Directorate of Health, Lubombo Spatial Development Initiative Malaria Program, Matola, Maputo Province, Mozambique; Division of Clinical Pharmacology, University of Cape Town, Cape Town, South Africa; Hunter New England Population Health, Newcastle, New South Wales, Australia; School of Public Health and Tropical Medicine, James Cook University, Townsville, Australia; Malaria Control Programme, Department of Health, Manzini, Swaziland; Malaria Control Programme, Mpumalanga Provincial Department of Health, Nelspruit, South Africa; Malaria Control Programme, KwaZulu-Natal Provincial Department of Health, Jozini, South Africa; National Health Directorate, Department of Epidemiology and Endemic Diseases, Ministry of Health, Maputo, Mozambique.

Abstract. The Lubombo Spatial Development Initiative is a joint development program between the governments of Mozambique, Swaziland, and South Africa, which includes malaria control as a core component of the initiative. Vector control through indoor residual spraying (IRS) was incrementally introduced in southern Mozambique between November 2000 and February 2004. Surveillance to monitor its impact was conducted by annual cross-sectional surveys to assess the prevalence of Plasmodium falciparum infection, entomologic monitoring, and malaria case notification in neighboring South Africa and Swaziland. In southern Mozambique, there was a significant reduction in $P$. falciparum prevalence after the implementation of IRS, with an overall relative risk of 0.74 for each intervention year $(P<0.001)$, ranging from 0.66 after the first year to 0.93 after the fifth intervention year. Substantial reductions in notified malaria cases were reported in South Africa and Swaziland over the same period. The success of the program in reducing malaria transmission throughout the target area provides a strong argument for investment in regional malaria control.

\section{INTRODUCTION}

The Lubombo Spatial Development Initiative (LSDI) is a joint program between the governments of Mozambique, Swaziland, and South Africa to develop the Lubombo region of eastern Swaziland, southern Mozambique (Maputo province), and northeastern KwaZulu-Natal into a globally competitive economic zone (Figure 1). The communities in this high malaria risk area include some of the poorest in the region, with high unemployment levels. ${ }^{1}$ There is evidence that malaria control is a necessary precursor to development in malaria-endemic areas. ${ }^{2}$ This contention is supported by well-documented negative impacts of malaria on tourism and agricultural development in the 1930s, before control measures were implemented. ${ }^{3}$ Thus, a malaria control program was initiated as a core component of the LSDI.

In July 1999, the heads of state of the three countries signed a protocol of understanding creating a platform for regional cooperation and delivery. In October 1999, a tri-national malaria program was launched at a ministerial level between the three countries.

The highest-risk malaria areas in South Africa and Swaziland border southern Mozambique, and there is ongoing migration between these neighboring areas. ${ }^{3}$ The malaria control program of the LSDI aimed to optimize the effectiveness of malaria control in the highest-risk malaria regions of South Africa and Swaziland bordering Mozambique, but placed primary emphasis on extending malaria control into southern Mozambique.

We report on malaria control activities from December 1999 to June 2005 in the LSDI areas of Mozambique, South Africa, and Swaziland and assess the impact of the control program on malaria prevalence, malaria incidence, and entomology in this region.

* Address correspondence to Immo Kleinschmidt, Medical Research Council, 491 Ridge Road, Durban 4091, South Africa. E-mail: ikleinschmidt@mrc.ac.za

\section{MATERIALS AND METHODS}

Interventions. Anopheles vector control through indoor residual spraying (IRS) with insecticide and parasite control through first-line treatment with artemisinin-based combination therapy (ACT) were the two key malaria control interventions implemented.

In Mozambique, twice annual IRS with bendiocarb insecticide (Bayer CropScience, Mannheim, Germany) at $400 \mathrm{mg} /$ $\mathrm{m}^{2}$ was introduced incrementally in five zones (1, 1A, 2, 2A, 3 ) in Maputo Province. Starting in Zone 1 (Figure 1) in November 2000, the program was extended to Zones $1 \mathrm{~A}$ and $2 \mathrm{~A}$ in February 2001, Zone 2 in October 2002, and Zone 3 in February 2004 (Table 1). In Zone 2A, IRS was interrupted from 2001 to 2002 because of resource constraints, but resumed in the second half of 2003 under a grant from the Global Fund against AIDS, Tuberculosis and Malaria. The five zones comprise an area of $\sim 21,000 \mathrm{~km}^{2}$, with a population of $\sim 800,000$, covering seven districts.

In Swaziland, IRS with dichloro-diphenyl-dicloroethylene (DDT; $2 \mathrm{~g} / \mathrm{m}^{2}$ ) had started in $1981 .{ }^{4}$ Spraying dates during the study period were September to December each year from 1999 to 2005. In South Africa, IRS with DDT started in $1946 .{ }^{5}$ In 1996, the policy changed to pyrethroid use. However, DDT was reintroduced in KwaZulu-Natal Province ${ }^{5}$ in 2000 after the detection of monooxygenase-based pyrethroid insecticide resistance, which led to program failure but which does not confer cross-resistance to DDT. ${ }^{6,7}$ Since 2001 , DDT IRS was conducted in October each year in both Mpumalanga and KwaZulu-Natal provinces. Pyrethroid insecticide continued to be used in homes with painted walls because of the visible residues of DDT on such surfaces. In KwaZulu-Natal, bendiocarb $\left(400 \mathrm{mg} / \mathrm{m}^{2}\right)$ was sprayed twice annually in homes with painted walls during the 2004/2005 malaria season.

All spraying was conducted using Hudson Xpert pumps (Hudson Manufacturing Co., Chicago, IL) with appropriate nozzles. Spraying personnel were trained in spraying techniques, safety measures, and personal protection equipment 


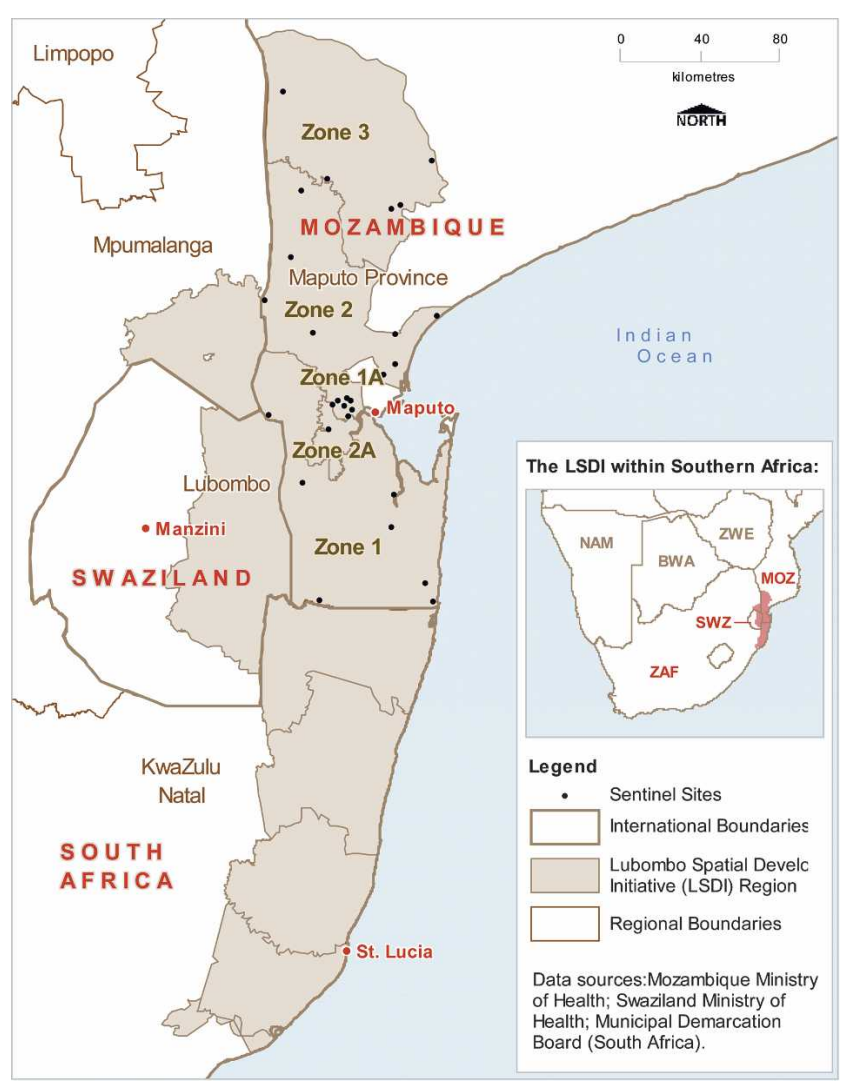

FIGURE 1. Map of the LSDI malaria control zones and location of sentinel sites. BWA, Botswana; MOZ, Mozambique; NAM, Namibia; ZAF, South Africa; SWZ, Swaziland; ZWE, Zimbabwe.

appropriate to the insecticide used. Given environmental concerns related to DDT use, the South African Endangered Wildlife Trust assisted in preparing training material and audited use, safety, and disposal of DDT.

Before the introduction of artemisinin-based combination therapy, chloroquine and sulfadoxine-pyrimethamine (SP) had been the first- and second-line treatments, respectively, in both Swaziland and Mozambique, whereas in South Africa, sulfadoxine-pyrimethamine had been the first-line treatment. In KwaZulu-Natal, artemether-lumefantrine was introduced in February 2001, whereas artesunate plus SP was introduced as first-line treatment in Mpumalanga Province in January 2003. ${ }^{8}$ The phased implementation of artesunate plus SP started in Zone 1 of Maputo Province in 2004, and in the Boane district (Zone 2A) in January 2005. Extension of this treatment policy to all zones will be completed during 2006.

Parasite prevalence surveys in Maputo Province, Mozambique. At each of 26 sentinel sites, cross-sectional parasite surveys were performed on a random sample of 120 individuals $\geq 2$ years of age. Sentinel sites were each divided into localities from which participants were selected to ensure as much geographical spread as feasible. Rapid diagnostic tests that detect histidine-rich protein 2 (HRP-2) antigen (ICT; Global Diagnostics, Cape Town, South Africa, and Kat Quick; Kat Medical, Johannesburg, South Africa) were used to assess prevalence of Plasmodium falciparum infection. Individuals who tested positive were treated, initially with chloroquine, and from 2001 with SP. In each zone, at least one survey was conducted in June before IRS intervention to pro- vide estimates of pre-spraying baseline prevalence of $P$. falciparum infection. Post-intervention surveys were conducted in June of each subsequent year. All age categories were sampled in December 1999, and subsequent surveys were confined to children 2 to $<15$ years of age.

The sentinel site specific sample size was determined to allow detection of a significant change at the $5 \%$ significance level, assuming a reduction in $P$. falciparum prevalence of at least $20 \%$ after intervention. Prevalence was calculated annually for each zone, and 95\% confidence intervals (CIs) were calculated using variance estimates that took account of clustering by sentinel site using the Rao and Scott correction, ${ }^{9}$ as implemented in the statistical software package STATA. ${ }^{10}$ Sentinel sites were considered the primary sampling unit. Logistic regression, allowing for complex survey designs, was performed to estimate the mean effect on prevalence of infection caused by each spray round, adjusted for differences in $P$. falciparum prevalence at baseline. To test for a secular time trend over the study period, a logistic regression model was used to compare baseline prevalence of infection in different years. This was possible because of the phased introduction of the intervention, thus providing baseline data over a range of years.

Malaria case notification data: South Africa and Swaziland. The number of malaria cases (confirmed microscopically or by antigen testing) for Swaziland and South Africa were obtained from their national Malaria Information Systems (MIS), because malaria is a notifiable disease in these countries. The MIS was designed to document all malaria cases notified by health facilities, and in the case of South Africa, includes actively detected cases during studies by field staff of malaria outbreaks or while conducting random household visits and in follow-up of confirmed cases. Although the South African MIS collects information on whether a case was "imported" or "local," this information was not consistently available for all study years, particularly when the case load was high. It was therefore decided not to use the data on imported cases for the purpose of this study.

Mosquito collection in Maputo Province, Mozambique. Mosquitoes were collected from 138 window traps that were fitted to selected houses at all sentinel sites. Houses with window traps were treated in the same way as other houses in the intervention area. Window traps were emptied daily, and mosquitoes were preserved in specimen jars that were collected monthly. Anophelines were morphologically classified and recorded as Anopheles (An). gambiae sensu lato (s.l.) and An. funestus s.l., ${ }^{11}$ initially at the South African Medical Research Council (MRC), and following training, at the LSDI center in Mozambique. Molecular analysis of specimens was undertaken at MRC laboratories in Durban. DNA was extracted from heads and thoraces of mosquitoes, ${ }^{12}$ and member species of the An. gambiae complex and of the An. funestus group were identified using polymerase chain reaction (PCR) techniques. ${ }^{13,14}$ PCR was also used to determine $P$. falciparum sporozoite status of all genetically identified $A n$. gambiae s.l. and An. funestus s.l. mosquitoes. ${ }^{11,15}$ Numbers of mosquitoes per trap per night were calculated for each vector species, both pre- and post-IRS. Using the species-specific estimated sporozoite prevalence, the number of infective mosquitoes per trap per night by species was calculated; the ratio of infective numbers per trap per night post-spraying, relative to pre-spraying, was defined as the relative transmis- 
TABLE 1

Prevalence of $P$. falciparum infection in children 2-14 years by zone and year estimated from prevalence surveys in the intervention area in Mozambique, with years pre-IRS results shaded (\%; sample surveyed [n]; 95\% CI)

\begin{tabular}{|c|c|c|c|c|c|c|c|c|}
\hline & Year & 1999 & 2000 & 2001 & 2002 & 2003 & 2004 & 2005 \\
\hline \multirow[t]{2}{*}{ Zone 1} & $\%(n)$ & $65 \%(597)$ & $60 \%(918)$ & $38 \%(807)$ & $22 \%(824)$ & $8 \%(792)$ & 7\% (839) & $4 \%(698)$ \\
\hline & {$[95 \% \mathrm{CI}]$} & [45-80\%] & [36-81\%] & [25-53\%] & [11-39\%] & {$[5-13 \%]$} & [5-11\%] & [3-6\%] \\
\hline \multirow[t]{2}{*}{ Zone $1 \mathrm{~A}$} & $\%(n)$ & & $88 \%(397)$ & $63 \%(592)$ & $36 \%(614)$ & $17 \%(714)$ & $21 \%(600)$ & $21 \%(599)$ \\
\hline & {$[95 \% \mathrm{CI}]$} & & [79-93\%] & [48-75\%] & [24-49\%] & [13-21\%] & [11-37\%] & {$[8-46 \%]$} \\
\hline \multirow[t]{2}{*}{ Zone 2} & $\%(n)$ & & & & $69 \%(955)$ & $34 \%(992)$ & $30 \%(954)$ & $20 \%(935)$ \\
\hline & {$[95 \% \mathrm{CI}]$} & & & & [58-79] & {$[25-45]$} & [20-42] & [13-30] \\
\hline \multirow[t]{2}{*}{ Zone 3} & $\%(n)$ & & & & & $70 \%(617)$ & $58 \%(579)$ & $33 \%(570)$ \\
\hline & {$[95 \% \mathrm{CI}]$} & & & & & [54-81] & {$[42-73]$} & [19-50] \\
\hline \multirow[t]{2}{*}{ Zone $2 \mathrm{~A}^{*}$} & $\%(n)$ & 73 (101) & 79 (120) & $32(130)$ & $51(117)$ & $59(118)$ & $39(120)$ & $23(114)$ \\
\hline & {$[95 \% \mathrm{CI}]$} & [62-82] & [70-86] & [23-43] & [38-64] & [49-69] & [28-52] & [15-34] \\
\hline
\end{tabular}

* In zone $2 \mathrm{~A}$, the intervention was interrupted from 2002 to 2003 .

sion index. The significance test comparing sporozoite prevalence in mosquitoes post-spraying with pre-spraying was carried out using generalized estimating equations, ${ }^{16}$ as implemented in STATA,${ }^{10}$ to allow for the correlation structure in the data.

Ethical considerations. Ethical approval for the study was obtained from the South African Medical Research Council and the Ministries of Health in Swaziland and Mozambique.

\section{RESULTS}

Plasmodium falciparum prevalence in Maputo Province, Mozambique. A total of 15,508 individuals were tested for parasitemia in the five zones of the Mozambican study area between December 1999 and June 2005, using rapid diagnostic tests (RDTs).

In 1999 in Zone 1, the average infection rate in children (age, 2-14 years) was $64 \%$ compared with $30 \%$ in adults (relative risk [RR], 2.12; 95\% CI, 1.87-2.41), with no evidence of heterogeneity in RRs between sites $(P=0.90)$. Prevalence surveys in Mozambique were subsequently restricted to children between 2 and $<15$ years of age.

Plasmodium falciparum prevalence estimated from prespray baseline surveys was $>60 \%$ for children $2-14$ years in all zones, regardless of the year during which the baseline survey was conducted (Table 1). A logistic regression model of prevalence of infection at baseline (pre-IRS) showed no significant difference in $P$. falciparum prevalence $(P=0.79)$ between baseline surveys undertaken from 1999 to 2003.

There was a decline in $P$. falciparum prevalence in each zone in each year after the introduction of IRS, except for in Zone 2A in 2002 and 2003, when IRS was interrupted (Table $1)$. The logistic regression model of prevalence of infection (excluding Zone 2A), with zone and number of years since baseline as explanatory variables, shows that prevalence at baseline was significantly higher in Zone 1A compared with Zone 1 (odds ratio [OR], 2.6; 95\% CI, 1.3-5.1). There was no significant difference in prevalence at baseline between Zone 1 and the remaining zones. There was a significant reduction in prevalence for each year after intervention across all zones (OR, 0.48/intervention year; 95\% CI, 0.42-0.56; $P<0.001$ ). There was no significant difference in the annual reduction in prevalence since baseline between different zones $(P=0.74$ for test for interaction between the effects of intervention years and zones).

In Zone 2A, average $P$. falciparum prevalence declined from $76 \%$ in the pre-spraying surveys of 1999 and 2000 to $33 \%$ after the first year of spraying, but rebounded to $59 \%$ by June 2003 after interruption of spraying. After the resumption of IRS, prevalence decreased steadily to $23 \%$ by June 2005 (Table 1).

Malaria cases notified in Swaziland and South Africa. The total number of notified confirmed malaria cases for Swaziland and South Africa decreased substantially between 1999/ 2000 and 2004/2005 (Table 2). The reductions were particularly large in those districts of KwaZulu-Natal, Swaziland, and Mpumalanga, which share borders with the intervention area in Mozambique.

Mosquito vector identification, abundance, and infection prevalence in Maputo Province, Mozambique. Anopheles arabiensis, An. merus, and An. quadriannulatus were the three member species of the An. gambiae complex genetically identified during the study period (Table 3). An. arabiensis accounted for $85 \%$ of identifications before IRS and $35 \%$ after IRS; An. merus increased to $58 \%$ after IRS. Five species of the An. funestus group were identified, the majority being An. funestus s.s. The relative numbers of An. gambiae s.l. and

TABLE 2

Notified confirmed malaria case totals for Swaziland and the two adjacent malaria-endemic provinces in South Africa for the period July 1999 to June 2003 and the percentage change in case numbers per malaria season in comparison with the baseline year of 1999/2000

\begin{tabular}{|c|c|c|c|c|c|c|}
\hline \multirow[b]{2}{*}{ Malaria season } & \multicolumn{2}{|c|}{ Swaziland } & \multicolumn{2}{|c|}{ KwaZulu-Natal } & \multicolumn{2}{|c|}{ Mpumalanga } \\
\hline & Cases & $\begin{array}{l}\text { Percent } \\
\text { change }\end{array}$ & Cases & $\begin{array}{l}\text { Percent } \\
\text { change }\end{array}$ & Cases & $\begin{array}{l}\text { Percent } \\
\text { change }\end{array}$ \\
\hline $1999 / 2000$ & 4,005 & & 41,077 & & 13,856 & \\
\hline 2000/2001 & 1,395 & -56 & 16,985 & -59 & 12,799 & -8 \\
\hline $2001 / 2002$ & 756 & -81 & 2,818 & -93 & 9,391 & -32 \\
\hline $2002 / 2003$ & 343 & -91 & 1,709 & -96 & 4,068 & -71 \\
\hline $2003 / 2004$ & 614 & -85 & 4,200 & -90 & 4,738 & -66 \\
\hline $2004 / 2005$ & 200 & -95 & 1,778 & -96 & 3,099 & -78 \\
\hline
\end{tabular}

A malaria season is the period from 1 July of 1 year to 30 June of the next. 
An. funestus s.l caught exiting window traps were substantially reduced after IRS. Only An. arabiensis, An. merus, and An. funestus s.s. were found to contain sporozoites; the prevalence of sporozoites decreased after spraying in all three species. The relative transmission index (the number of infective mosquitoes per trap per night relative to pre-intervention) was 0.002 for An. arabiensis, 0.067 for An. merus, and 0.016 for An. funestus s.s.

\section{DISCUSSION}

Before initiating vector control activities in Mozambique, $P$. falciparum malaria prevalence among children was high in all zones studied, with average infection by zone ranging from $62 \%$ to $86 \%$. These results were similar to prevalence surveys conducted during the 1937/38 season in 10 areas in Zone 1, which showed high parasite prevalence with an average of $86 \%$ of children 1 to $<15$ years of age infected. ${ }^{17}$ Pre-malaria control parasite prevalence in KwaZulu-Natal in 1932 was similar to the baseline data from Mozambique reported in this study, with $73 \%$ infection in children up to 15 years of age and $42 \%$ in adults. ${ }^{3}$ Age-specific $P$. falciparum infection prevalence in Zone 1 was as expected in high-risk malaria areas without malaria control programs in that infection in the $<15$-year-old categories was approximately double that found in the older age groups, reflecting partial immunity acquired with increasing age after repeated malaria infections. ${ }^{18}$

Indoor residual spraying using carbamate insecticide in Mozambique was associated with a statistically significant reduction in parasite prevalence in all zones with an OR of 0.48 (95\% CI, 0.42-0.56) per intervention year. Because these reductions were significant across all zones, and ACTs were only introduced in two of the five zones in the last year of this study, the overall reduction in $P$. falciparum prevalence in southern Mozambique was largely attributable to IRS. The implementation of ACTs in South Africa is considered an important contributor to improved malaria control through improved cure rates and decreased gametocyte carriage. ${ }^{8}$ This study has shown the benefits of reducing transmission intensity by establishing vector control before introducing definitive diagnosis, followed by ACTs as first-line treatment.

Entomologic surveillance showed radical reductions in numbers of mosquitoes caught in window traps after the introduction of IRS for all three vectors that were implicated in malaria transmission. In An. arabiensis and An. funestus s.s., the decrease in sporozoite prevalence after IRS was statistically significant. Although the reduction in sporozoite prevalence was not statistically significant in An. merus, the majority of post-spraying sporozoite-positive $A n$. merus specimens were found during the first intervention year. Table 3 shows that relative abundance of An. merus was low before IRS was introduced, confirming that it was not a major vector in the study area. While this species constituted a larger proportion of all An. gambiae s.l. specimens captured after spraying than before spraying, this is mainly a reflection of the disproportionately large decline in An. arabiensis numbers after the introduction of IRS.

This is the first application using the carbamate insecticide bendiocarb in a large-scale house spraying program in Africa, and results are comparable with historical data using other insecticides. This includes data for the same area of southern Mozambique over the period 1961-1969 when all houses were sprayed annually with DDT and parasite prevalence rates were reduced to $<5 \%$ in all areas, an effect that was sustained for the duration of the campaign. ${ }^{19}$ Similar parasite prevalence reductions were obtained in the Pare-Tavete area of Tanzania after house spraying with dieldrin, ${ }^{20}$ in the Kisumu area of Kenya after application of fenitrothion, ${ }^{21}$ and in Zanzibar and Pemba in Tanzania after DDT house spraying. ${ }^{22} \mathrm{~A}$ more recent example is that of Bioko, Equatorial Guinea, where prevalence of infection was reduced from $46 \%$ to $32 \%$ after the first round of spraying. ${ }^{23}$ All of these interventions recorded significant reductions in parasite prevalence after

TABLE 3

Identification of member species, abundance, and transmission rates of malaria-transmitting mosquitoes caught in window traps in intervention areas of Mozambique, $\S 1999-2005$

\begin{tabular}{|c|c|c|c|c|c|}
\hline \multicolumn{3}{|c|}{ An. gambiae s.l. } & \multicolumn{3}{|c|}{ An. funestus s.l. } \\
\hline & Pre-IRS & Post-IRS & & Pre-IRS & Post-IRS \\
\hline Total number of mosquitoes collected & 5,577 & 969 & & 8,830 & 2,107 \\
\hline Number tested by PCR & 903 & 721 & & 818 & 575 \\
\hline Percent An. arabiensis & 85 & 35 & Percent An. funestus s.s. & 95 & 65 \\
\hline Percent An. merus & 11 & 58 & Percent An. rivulorum & 1 & 24 \\
\hline \multirow{3}{*}{ Percent An. quadriannulatus } & 4 & 7 & Percent $A n$. vaneedeni & 0 & 3 \\
\hline & & & Percent $A n$. parensis & 1 & 2 \\
\hline & & & Percent An. leesoni & 3 & 6 \\
\hline
\end{tabular}

\begin{tabular}{|c|c|c|c|c|c|c|}
\hline \multirow[b]{3}{*}{ Total estimated } & Pre-IRS & Post-IRS & Pre-IRS & Post-IRS & \multicolumn{2}{|c|}{ An. funestus s.s. } \\
\hline & \multicolumn{2}{|c|}{ An. arabiensis } & \multicolumn{2}{|c|}{ An. merus } & Pre-IRS & Post-IRS \\
\hline & 4,740 & 339 & 613 & 562 & 8,389 & 1,370 \\
\hline Mean/trap/100 nights (density) & 14.9 & 0.2 & 1.9 & 0.4 & 26.3 & 0.9 \\
\hline Sporozoite prevalence, $\%(n), p$ & $7.1(758)$ & $\begin{array}{l}0.8(239) \\
p=0.002\end{array}$ & $2.2(91)$ & $\begin{array}{l}0.7(413) \\
p=0.25\end{array}$ & $4.7(773)$ & $\begin{array}{l}2.2(367) \\
p=0.04\end{array}$ \\
\hline Transmission index* & 1.058 & 0.002 & 0.04 & 0.003 & 1.236 & 0.020 \\
\hline Transmission index relative to baseline & 1.00 & 0.002 & 1 & 0.067 & 1.0 & 0.016 \\
\hline
\end{tabular}

$\S$ Excluding zone $2 \mathrm{~A}$, where the intervention was interrupted.

* The estimated number of infective mosquitoes exiting window traps. 
the initial spraying round, with the mosquito vector $A n$. funestus s.l. reduced to negligible levels or eliminated and $A n$. gambiae s.l. populations reduced to very low numbers. In our study, the substantial reductions in An. funestus s.l. and An. gambiae s.l. populations after IRS confirm the effectiveness of large-scale vector control by sustained indoor residual spraying in Africa.

Conteh and others ${ }^{24}$ compared the cost and cost effectiveness of using carbamates for vector control in Mozambique with the findings of costing studies of other IRS programs and concluded that the cost per person covered per year in the LSDI was mid-to-high in the ranking of theses studies, but showed how overall cost would decrease if cheaper insecticides such as DDT were substituted. Both IRS and insecticide treated nets (ITNs) have been shown to be attractive malaria interventions in low- and middle-income countries. ${ }^{24,25}$

A substantial proportion of malaria cases in Swaziland and South Africa are annually classified as imported, the majority originating in Mozambique. ${ }^{26}$ Ngxongo $^{27}$ found that $69 \%$ of the KwaZulu Natal malaria cases were detected in the district bordering Mozambique. Thus, it is not surprising that the reduction in prevalence of the previously uncontrolled reservoir of infection in southern Mozambique would impact on transmission in the adjoining areas, particularly KwaZuluNatal and Swaziland. This is particularly supported by the $95 \%$ reduction in malaria cases recorded in Swaziland, where no major changes in malaria control occurred during the study period (Table 2). In Mpumalanga Province in South Africa, which only partially borders Zone 1, malaria case notifications decreased by $78 \%$.

The LSDI regional malaria control initiative has markedly reduced $P$. falciparum malaria prevalence among children in southern Mozambique and reduced malaria transmission in neighboring areas with established malaria control. This study shows how a regional approach to communicable disease control can have significant public health benefits in terms of decreased human morbidity and averted treatment costs. It also confirms the effectiveness of large-scale vector control in endemic malaria areas. Based on these results, additional funding has been secured to extend malaria vector control in Mozambique to a further area of $75,000 \mathrm{~km}^{2}$, which will result in a contiguous area of $>195,000 \mathrm{~km}^{2}$ inhabited by $>6$ million people in the three countries being protected. There is little doubt that this regional approach will contribute to "Rolling Back Malaria" and reduce the economic burden of malaria across all three countries, providing a strong argument for ongoing investment by these neighboring countries in regional malaria control.

Received July 27, 2006. Accepted for publication September 5, 2006.

Acknowledgments: This program would not have been possible without political, technical, and staff support of the Ministries of Health of Mozambique and Swaziland, the South African Department of Health, the University of Cape Town, and the Medical Research Council of South Africa. The South African Ministry of Tourism and Andrew Zaloumis, Manager of the LSDI, are acknowledged for consistent support and encouragement, without which this project would not have succeeded.

Financial support: The authors acknowledge the financial support of the South African Business Trust, B.H.P. Billiton, the South African Government, the Mozambique Ministry of Health, the South African Department of Science and Technology, and the Global Fund for
AIDS, Tuberculosis and Malaria. The South African Innovation Fund contributed toward the Decision Support System for the LSDI.

Authors' addresses: Brian L. Sharp, Medical Research Council, 491 Ridge Road, Durban, South Africa, Telephone: 27-31-20304700, Fax: 27-31-203-4701, E-mail: sharpb@mrc.ac.za. Immo Kleinschmidt, Medical Research Council, 491 Ridge Road, Durban 4091, South Africa, Telephone: 27-31-203-4806, Fax: 27-31-203-4704, E-mail: ikleinschmidt@mrc.ac.za. Elizabeth Streat, Maputo Province Directorate of Health, Lubombo Spatial Development Initiative Malaria Program, Matola, Maputo Province, Mozambique. Rajendra Maharaj, Medical Research Council, 491 Ridge Road, Durban 4091, South Africa. Karen I. Barnes, Division of Clinical Pharmacology, University of Cape Town, Cape Town, South Africa. David N. Durrheim, Hunter New England Population Health, Newcastle, New South Wales, Australia. Frances C. Ridl, Medical Research Council, 491 Ridge Road, Durban 4091, South Africa. Natashia Morris, Medical Research Council, 491 Ridge Road, Durban 4091, South Africa. Ishen Seocharan, Medical Research Council, 491 Ridge Road, Durban 4091, South Africa. Simon Kunene, Malaria Control Programme, Department of Health, Manzini, Swaziland. Jacobus J.P. la Grange, Malaria Control Programme, Mpumalanga Provincial Department of Health, Nelspruit, South Africa. Jotham D. Mthembu, Malaria Control Programme, KwaZulu-Natal Provincial Department of Health, Jozini, South Africa. Francois Maartens, Medical Research Council, 491 Ridge Road, Durban 4091, South Africa. Carrin Martin, Medical Research Council, 491 Ridge Road, Durban 4091, South Africa. Avertino Barreto, National Health Directorate, Department of Epidemiology and Endemic Diseases, Ministry of Health, Maputo, Mozambique.

\section{REFERENCES}

1. Sharp BL, Craig MH, Mnzava A, Curtis B, Maharaj R, Kleinschmidt I, 2001. Review of malaria in South Africa. Available at http://www.hst.org.za/uploads/files/chapter18_00.pdf. Accessed October 19, 2006.

2. Gallup JL, Sachs JD, 2001. The economic burden of malaria. Am J Trop Med Hyg 64(1-2 Suppl): 85-96.

3. Sharp BL, le Sueur D, 1996. Malaria in South Africa-the past, the present and selected implications for the future. $S$ Afr Med $J$ 86: 83-89.

4. Mabaso MLH, Sharp BL, le Sueur D, Lengeler C, 2004. Historical review of malaria control in southern African with emphasis on the use of indoor residual house spraying. Trop Med Int Health 9: 846-856.

5. Maharaj R, Mthembu DJ, Sharp BL, 2005. The impact of the reintroduction of DDT on malaria transmission in KwaZuluNatal, South Africa. S Afr Med J 95: 871-874.

6. Hargreaves K, Koekemoer LL, Brooke BD, Hunt RH, Mthembu J, Coetzee M, 2000. Anopheles funestus resistant to pyrethroid insecticides in South Africa. Med Vet Entomol 14: 181-189.

7. Casimiro S, Coleman M, Moholai P, Hemingway J, Sharp B, 2006. Insecticide resistance in Anopheles funestus (Diptera: Culicidae) from Mozambique. J Med Entomol 43: 267-275.

8. Barnes K, Durrheim D, Little F, Jackson A, Mehta U, Allen E, Dlamini S, Tsoka J, Bredenkamp B, Mthembu J, White N, Sharp B, 2005. Effect of artemether-lumefantrine policy and improved vector control on malaria burden in KwaZulu-Natal, South Africa. PLoS Med 2: 1-12.

9. Rao JNK, Scott AJ, 1981. The analysis of categorical data from complex sample surveys: chi-squared tests for goodness of fit and independence in two-way tables. J Am Stat Assoc 76: 221230.

10. StataCorp, 2005. Stata Statistical Software: Release 9. College Station, TX: StataCorp LP.

11. Gillies MT, Coetzee M, 1987. A Supplement to the Anophelinae of Africa South of the Sahara. Johannesburg: The South African Institute for Medical Research.

12. Boom R, Sol CJA, Salimans MM, Jansen CL, Wertheim-Van Dillen PME, Van Der Noordaa J, 1990. Rapid and simple method for purification of nucleic acids. J Clin Microbiol 28: 495-503.

13. Scott A, Brogdon WG, Collins FH, 1993. Identification of single 
specimens of the Anopheles gambiae group by polymerase chain reaction. Am J Trop Med Hyg 49: 520-529.

14. Koekemoer LL, Kamau L, Hunt RH, Coetzee M, 2002. A cocktail polymerase chain reaction assay to identify members of the Anopheles.funestus (Diptera: Culicidae) group. Am J Trop Med Hyg 6: 804-811.

15. Snounou G, Viriyakosol S, Zhu XP, Jarra W, Pinheiro L, do Rosario VE, Thaithong S, Brown KN, 1993. High sensitivity of detection of human malaria parasites by the use of nested polymerase chain reaction. Mol Biochem Parasitol 61: 315-320.

16. Zeger SL, Liang KY, Albert PS, 1988. Models for longitudinal data: A generalised estimating equation approach. Biometrics 44: 1049-1060.

17. Martins P, 1941. Contribuicao para a Confeccao da Carta Sezonatica da Colonia de Mocambique. Maputo, Mozambique: Primeiro Congresso Medico De Lourenco Marques.

18. Baird JK, Masbar S, Basri H, Tirtokusumo S, Subianto B, Hoffman SL, 1998. Age-dependent susceptibility to severe disease with primary exposure to Plasmodium falciparum. J Infect Dis 178: 592-595.

19. Schwalbach JFL, De La Maza MCR, 1974. A Malaria em Mocambique (1937-1973). Republica Populat De Mocambique: Ministerio Da Saude, Instituto Nacional De Saude.

20. Draper CC, Smith A, 1960. Malaria in the pare area of Tanganyika. Effects of 3 years of spraying of huts with dieldrin. Trans $R$ Soc Trop Med Hyg 54: 342.
21. Payne D, Grab B, Fontaine RE, Hempel JHG, 1976. Impact of control measures on malaria transmission and general mortality. Bull WHO 54: 369-377.

22. Kouznetsov R, 1977. Malaria control by application of residual insecticides in tropical Africa and its impact on community health. Trop Doct 7: 81-91.

23. Kleinschmidt I, Sharp B, Benavente L, Schwabe C, Torrez M, Kuklinski J, Morris N, Raman J, Carter J, 2006. Reduction in infection with $P$. falciparum one year after the introduction of malaria control interventions on Bioko Island, Equatorial Guinea. Am J Trop Med Hyg 74: 972-978.

24. Conteh L, Sharp BL, Streat E, Barreto A, Konar S, 2004. The cost and cost-effectiveness of malaria vector control by residual insecticide house-spraying in southern Mozambique: A rural and urban analysis. Trop Med Int Health 9: 125-132.

25. Goodman CA, Coleman PG, Mills AJ, 2000. Economic Analysis of Malaria Control in Sub-Saharan A frica. Geneva: Global Forum for Health Research.

26. Sharp BL, Ngxongo S, Botha S, Ridl F, le Sueur D, 1988. An analysis of 10 years retrospective data from the KwaZulu areas of Natal. S Afr J Sci 84: 102-106.

27. Ngxongo SM, 1993. The epidemiology of malaria in KwaZulu, 1980-1991. MSc thesis, University of Natal, Durban, South Africa. 